\title{
The analysis to the latest changes in NCCN Guidelines of Central Nervous System Cancers about low-grade gliomas and glioblastoma
}

Tianyu Wang ${ }^{1,2,3}$, Xiong Xiao ${ }^{1,2,3}$ and Nan $\mathrm{ji}^{1,2,3^{*}}$

\begin{abstract}
Gliomas are the most common primary tumors of the central nervous system, around $70 \%$ of the malignant brain tumors are gliomas. In the NCCN Guideline Ver.1 2015, the assessment before systemic treatment, treatment principles and prognosis factors of gliomas has significantly changed based on the researches up to date, we try to analyze the reason and the effect of these changes. The most important change is the reintroduction of PCV chemotherapy in systemic treatment, which narrows the gap of prognosis between WHO II and III gliomas. Other changes including the assessment before systemic treatment, usage of RT and the promotion of evidence level about the Tumor Treating Fields.
\end{abstract}

Keywords: Glioma, Glioblastoma, NCCN Guideline of Central Nervous System Cancers, PCV

\section{Background}

The simple term of glioma consists of many different types of tumors that arise from the neuroepithelial cell in central nervous system, which vary greatly in their histology, genetics, and clinical outcome. Basically, they are divided into 4 grades by WHO 2007 Classificaton of Tumors of the CNS. Despite of the Grade I gliomas which are considered to be benign, Grade II-IV are all malignant. Gliomas are the most common primary tumors of the central nervous system, around $70 \%$ of the malignant brain tumors are gliomas, with a annually morbidity of $6 /$ 100000 in the United States [1]. Besides grading, gliomas are further classified into pathological subtypes of which the most common include astrocytomas and oligodendrogliomas. Grade II astrocytomas and oligodendrogliomas can progress to grade III anaplastic astrocytomas and anaplastic oligodendrogliomas, respectively. Glioblastomas (GBMs) are grade IV astrocytomas including mainly "primary GBMs" (95 \%) and "secondary GBMs (5\%)" that have progressed from clinically discernible grade II or III astrocytomas [2].

\footnotetext{
*Correspondence: neurochina@gmail.com

'Department of Neurosurgery, Beijing Tiantan Hospital, Capital Medical University, 6 Tian Tan Xi Li, Beijing 100050, China

${ }^{2}$ Beijing Key Laboratory of Brian Tumor, Beijing 100050, China

Full list of author information is available at the end of the article
}

In the latest NCCN Guidelines of Central Nervous System Cancers (Version1, 2015), the assessment before systemic treatment, treatment principles and prognosis factors of gliomas has significantly changed based on the researches up to date, we try to analyze the reason and the effect of these changes as below.

\section{Main text}

1. The use of PCV treatment in gliomas.

As is mentioned in the Guidelines, chemotherapy is not a traditional mainstay of upfront treatment for low-grade gliomas. There are some data that support temozolomide as adjuvant therapy, and it is included as a category $2 \mathrm{~B}$ recommendation in 2004 based on non-uniform panel consensus.

Since the early 1990s, initial therapy for newly diagnosed anaplastic oligodendrogliomas $(\mathrm{AO})$ has become procarbazine, lomustine (CCNU), and vincristine (PCV), with or without radiotherapy, in many centers [3]. But some of the early trail shows that temozolomide shows a better curative effect and do no good to overall survival [4-9]. Based on the final report of RTOC 9402 date back to 2013, situation has changed a little. Chemoradiotherapy, especially $\mathrm{PCV}+\mathrm{RT}$, may be a highly effective treatment 
for patients with codeleted $\mathrm{AO} / \mathrm{AOA}$, but PCV plus RT cannot emerge as the new standard of care, because temozolomide is also effective for $\mathrm{AO} / \mathrm{AOA}$, and $\mathrm{PCV}$ is more toxic [10]. The complete dramatical change turned out in 2014 when the long-term follow-up of the RTOG 9802 trial was published. Comparing 54 Gy of radiotherapy (RT) with the same RT followed by adjuvant procarbazine, $\mathrm{CCNU}$, and vincristine (PCV) chemotherapy in high-risk low-grade glioma shows a major increase in survival after adjuvant PCV chemotherapy. Median overall survival increased from 7.8 to 13.3 years, with a hazard ratio of death of 0.59. This increase in survival was observed despite the fact that $77 \%$ of patients who progressed after RT alone received salvage chemotherapy. With this outcome, RT + PCV is now to be considered standard of care for low-grade glioma requiring postsurgical adjuvant treatment [11].

However, a number of regimens including PCV and temozolomide are currently considered acceptable for recurrence or progressive disease and confered survival benefits in the long term. And it is for sure that patients with low-grade oligodendrogliomas, especially those with 1p/19q deletions, may represent favorable candidates for chemotherapy in light of good response rates, this has never been prospectively determined, which makes PCV treatment now has a category 1 recommendation in the 2015 version [12-20].

The classic PCV treatment including procarbazine, lomustine (CCNU), and vincristine, and the usage is below:

$1^{\text {st }}$ day: CCNU $110 \mathrm{mg} / \mathrm{m}^{2}$, Po.

$8^{\text {th }}$ day and $29^{\text {th }}$ day: vincristine $1.4 \mathrm{mg} / \mathrm{m}^{2}$, iv.

$8^{\text {th }}$ day to $21^{\text {st }}$ day: procarbazine $60 \mathrm{mg} / \mathrm{m}^{2}$ per day, Po. And the course of treatment is 6 weeks.

Cyclohexyl nitrosourea (CCNU), a cell cycle non-specific medicine, is mostly sensitive to cells at the G1-S boundary or early $\mathrm{S}$ phase, and has also inhibition to G2 phase. After the product enters into the human body, its elements breaks into two parts from ammonia formamide bond: one part is the chlorine ethylamine, dissociaties chlorine to form ethylene carbocation, gives play to the role of alkylation, resulting in DNA strand breaks, RNA and protein by alkylation, which is mainly related to the anti-tumor effect; the other is carbamoyl portion, converts to isocyanate, or ammonia and then gives play to the role of ammonia formylation, reacting mainly with proteins, especially with lysine acid terminal amino. It is believed that this is mainly related to bone marrow toxicity, ammonia formylation can also destroy some enzyme protein, so DNA by alkylation damage is relatively difficult to repair, which contribute to anti-cancer effect. Although this product has the effects of alkylating agentt, but has no cross-resistance with the general alkylating agent, so also does with vincristine, procarbazine and Antimetabolite.

The function of vincristine is related to the concentration. At low concentrations, the product is combined with the point of low affinity tubulin, and due to space barrier and other factors, inhibit tubulin polymerization. At high concentration, the product is combined with high affinity points on tubulin, making the microtubules gathered together and forming a kind of crystallization. PCV program primarily make use of the characteristics of its low concentration.

In vivo, procarbazine is oxidized by erythrocytes and liver microsomal enzymesin to metabolite of azo procarbazine with antitumor effect, by the end of the N-methyl transmethylation, the methyl is transferred to 7 of guanine and 1 of adenine and alkylated. Furthemore, the methyl can also be transferred to the tRNA, which inhibiting not only synthesis of DNA and RNA, but also protein synthesis.

The recommendation to the PCV treatment has changed the guideline to a great extent. First of all, the prognosis importance and the guiding function of the biomarkers in gliomas would significantly change due to the treatment of PCV, considering it has a totally different theory comparing to the temozolomide. Meanwhile, the combination of RT and PCV chemotherapy has a more stable prognosis, regardless of the preoperative state of the patients, which lead to the other change in the Guideline Ver.1 2015. Moreover, PCV can narrow the gap between the prognosis of WHO II and WHOIII gliomas, which means a better curative effect than temozolomide. And the most important is that because of the treatment of PCV and RT is not so limited comparing to the temozolomide, maximal safe resection or subtotal resection would become the most important prognosis index. Taking these into consideration, the treatment of PCV maybe the most important change in the Guideline Ver.1 2015.

2. The change to the classification of low risk and high risk patients in low-grade gliomas and glioblastoma

Beside the change to the systemic therapy, the classification of preoperative state in patients has changed. The following are used to be considered as low-risk features in the past version of guideline: age $<40$ years, Karnofsky Performance Status (KPS) $\geq 70$, minor or no neurologic deficit, ologodendroglioma or mixed oligoastrocytoma, tumor dimension $<6 \mathrm{~cm}, 1 \mathrm{p} / 19 \mathrm{q}$ codeleted, and IDH 1 or 2 mutated. Otherwise would be high-risk. But now, the classification has been simplified to only considering resection range and the age. Considering the background that the enormous development of the Precision Medicine, this simplification may be very confusing. 
Partly, we think the confusing change to classification is related to a series of trails, which shows that the systemic therapy, especially PCV $+\mathrm{RT}$, would benefit patients in the long term [21]. By this means, patients who are treated with gross total resection (GTR) would benefit much more than those who are not. Such change can also be found in the treatment and prognosis to the glioblastomas. As is mentioned in the Guideline, age, rather than KPS is a key option in the decision tree branch points. The complex assessment before systemic treatment including biomarkers and neurologic deficit is abandoned, which makes it easier to evaluate the prognosis of patients and to choose the therapeutic regimen. This change means operation has turn into the most important factor in prognosis, whether the preoperative state of the patients is good or not. By this means, surgery may become the most important treatment and GTR can never be emphasized too much.

However, the changes in classification do not mean the recession of molecular pathology. In fact, in the trails ROTC 9802, studies on molecular correlates associated with response are still lacking [11]. That means further researches should be run to reevaluate the prognosis function of IDH1, TRET, EGFR, 1p/19q and p53 under the treatment of PCV + RT. In a similar way, KPS and preoperative state are also should be re-evaluated. It is certain that it would be an enormous variation to the prognostic values of most of the molecular biomarkers.

\section{Other changes in Guideline}

RT alone after operation is no longer an option of treatment. A series of trails show that patients of low-grade and high-grade gliomas would benefit from RT + chemotherapy [22-24], which means chemotherapy raises its importance. Still, thanks to the use of PCV treatment.

Meanwhile, the dose of RT for high-grade gliomas has changed, the recommended dose used to be 60Gy in 1.8 to 2.0Gy fractions, now it is $2.0 \mathrm{~Gy}$ fractions only. Which means a more focused RT program is recommended.

Moreover, the evidence level of alternating electric field therapy has been lifted from category 3 to category 2B. It is based on the results of a clinical trial that randomized 237 patients to Tumor Treating Fields (TTF) or chemotherapy. The results show the similar survival in the two arms, and TTF therapy was associated with lower toxicity and improved quality of life [25].

\section{Predictions for the development of the Guideline}

The GTR will own a higher position in the treatment of gliomas, which means, with the development of systemic treatment, neurosurgery operation will getting increasinglyimportant than before. And the assessment before systemic treatment will focus on resection and age, KPS will be less valued. Considering the development of systemic therapy, this trend is definitely continue in the next version of Guideline and will affect clinical strategy to a great extent.

Due to the reintroduction of PCV, most of the biomarkers prognosis function would change a lot, on account of they are used to be mainly connected with RT and Temozolmide. Though they are less linked with the assessment before systemic treatment, biomarkers which are the basement of the Precision Medicine, will play an increasingly - important role in the guideline to conduct the prognosis and treatment.

\section{Conclusions}

NCCN Guidelines of Central Nervous System Cancers about low-grade gliomas and glioblastoma Ver.1 2015 shows a trend of simplification of the assessment before systemic treatment and emphasize the importance of GTR and systemic treatment including PCV and RT. In a word, less is more.

\section{Abbreviations}

AO: Anaplastic oligodendrocytoma; AOA: Anaplastic oligodendroastrocytoma; CCNU: Lomustine; CNS: Central nervous system; EGFR: Endothelial growth factor receptor; GBM: Glioblastoma; GTR: Gross total resection; IDH: Isocitrate dehydrogenase; KPS: Karnofsky Performance Status; NCCN: National Comprehensive Cancer Network; PCV: Procarbazine and lomustine and vincristine; RT: Radiotherapy; TRET: Telomerase reverse transcriptase; TTF: Tumor treating fields; WHO: World Health Organiztion..

\section{Competing interests}

The authors declare that they have no competing interests.

\section{Authors' contributions}

TW and XX carried out the literature review and drafted the manuscript. NJ contributed to the design and revision of the manuscript. All authors read and approved the final manuscript.

Authors' information

Not applicable.

\section{Acknowledgements}

This project was supported by National Key Technology Research and Development Program of the Ministry of Science and Technology of China (2013BAI09B03)

\section{Author details}

${ }^{1}$ Department of Neurosurgery, Beijing Tiantan Hospital, Capital Medical University, 6 Tian Tan Xi Li, Beijing 100050, China. ${ }^{2}$ Beijing Key Laboratory of Brian Tumor, Beijing 100050, China. ${ }^{3}$ China National Clinical Research Center for Neurological Diseases, Beijing, China.

Received: 22 August 2015 Accepted: 21 September 2015

Published online: 05 November 2015

\section{Reference}

1. Ricard D, Idbaih A, Ducray F, Lahutte M, Hoang-Xuan K, Delattre JY. Primary brain tumours in adults. Lancet. 2012;379(9830):1984-96.

2. Louis DN, Ohgaki H, Wiestler OD, Cavenee WK, Burger PC, Jouvet A, et al. The 2007 WHO classification of tumours of the central nervous system. Acta neuropathologica. 2007;114(2):97-109.

3. Levin VA, Silver $\mathrm{P}$, Hannigan J, Wara WM, Gutin PH, Davis RL, et al. Superiority of post-radiotherapy adjuvant chemotherapy with CCNU, procarbazine, and vincristine (PCV) over BCNU for anaplastic gliomas: NCOG 6 G61 final report. Int J Radiat Oncol Biol Phys. 1990;18:321-4. 
4. Keasari S, Scgiff D, Drappatz J, LaFrankie D, Doherty L, Macklin EA, et al. Phase II study of protracted daily temozolomide for low-grade gliomas in adults. Clin Cancer Res. 2009;15:330-7.

5. Pouratian N, Gasco J, Sherman JH, Shaffrey ME, Schiff D. Toxicity and efficacy of protracted low dose temozolomide for the treatment of lowgrade gliomas. J Neurooncol. 2007;82:281-8.

6. Shaw EG, Wang M, Coons SW, Brachman DG, Buckner JC, Stelzer KJ, et al. Randomized trial of radiation therapy plus procarbazine, lomustine, and vincristine chemotherapy for supratentorial adult low-grade gliom:initial results of RTOG 9802. J Neurooncol. 2012;30:3065-70.

7. Chamberlain MCDOESRTOG. 9802 change practice with respect to newly diagnosed low-grade glioma? J Neurooncol. 2013;31:652-3.

8. van den Bent MJ, Jaeckle K, Baumert B, Wick W. BRTOG 9802:good wines need aging. J Neurooncol. 2013;31:653-4.

9. Perry JR, Rizek P, Cashman R, Morrison M, Morrison T. Temozolomide rechallenge in recurrent malignant glioma by using a continuous temozolomide schedule:the "rescue" approach. Cancer. 2008;113:2152-7.

10. Cairncross G, Wang M, Shaw E, Jenkins R, Brachman D, Buckner J, et al. Phase III Trial of Chemoradiotherapy for Anaplastic Oligodendroglioma: Long-Term Results of RTOG 9402. J Clin Oncol. 2013;31(3):337-43.

11. van den Bent MJ. Practice changing mature results of RTOG study 9802: another positive PCV trial makes adjuvant chemotherapy part of standard of care in low-grade glioma. Neuro-Oncology. 2014;16(12):1570-4.

12. Massimino M, Spreafico F, Riva D, Biassoni V, Poggi G, Solero C, et al. A lower-dose,lower-toxicity cisplatin-etoposide regimen for childhood progressive low-grade glioma. J Neurooncol. 2010;100(1):65-71.

13. Moghrabi A, Friedman HS, Ashley DM, Bottom KS, Kerby T, Stewart E, et al. Phase II study of carboplatin(CBDCA) in progressive low-grade gliomas. Neurosurg Focus. 1998;4:e3.

14. Brandes AA, Basso U, Vastola F, Tosoni A, Pasetto LM, Jirillo A, et al. Carboplain and teniposide as third-line chemotherapy in patients with recurrent oligodendroglioma or oligoastasrocytoma:a phase II study. Ann Oncol. 2003;14:1727-31.

15. Hoang-Xuan K, Capelle L, Kujas M, Taillibert S, Duffau H, Lejeune J, et al. Temozolomide as initial treatment for adults with low-grade oligodendrogliomas or oligoastasrocytomas and correlation with chromosome 1p deletions. J Clin Oncol. 2004;22:3133-8.

16. Kaloshi G, Benouaich-Amiel A, Diakite F, Taillibert S, Lejeune J, LaigleDonadey F, et al. Temozolomide for low-grade gliomas:predictive impact of 1p/19q loss on response and outcome. Neurology. 2007:68:1831-6.

17. Buckner JC, Gesme Jr D, O'Fallon JR. Phase II trial of procarbazine, lomustine, and vincristine as initial therapy for patients with low-grade oligodendroglioma or oligoastasrocytoma:efficacy and associations with chromosomal abnormalities. J Clin Oncol. 2003;21:251-5.

18. Cairncross G, Macdonald D, Ludwin S, Lee D, Cascino T, Buckner J, et al. Chemotherapy for anaplastic oligodendroglioma. National Cancer Institute of Canada Clinical Trials Group. J Clin Oncol. 1994:12:2013-21.

19. Ino Y, Betensky RA, Zlatescu MC, Sasaki H, Macdonald DR, StemmerRachamimov AO, et al. Molecular subtypes of anaplastic oligodendroglioma. Clin Cancer Res. 2001;7:839-45.

20. van den Bent M, Chinot OL, Cairncross JG. Recent developments in the molecular characterization and treatment of oligodendroglial tumors. Neuro oncol. 2003:5:128-38.

21. Buckner JC, Pugh SL, Shaw EG, Gilbert MR, Barger G, Coons S, et al. Phase III study of radiation therapy (RT) with or without procarbazine, $C \mathrm{CNU}$, and vincristine (PCV) in low-grade glioma: RTOG 9802 with Alliance, ECOG, and SWOG. J Clin Oncol. 2014:32:5s. abstract \#2000.

22. Medical Research Council Brain Tumor Working Party. Randomized trial of procaibazine, lomustine, and vincristine in the adjuvant treatment of highgrade astrocytoma: a Medical Research Council trial. J Clin Oncol. 2001;19:509-18.

23. Stewart LA. Chemotherapy in adult high-grade glioma: a systematic review and meta-analysis of inndividual patient data from 12 randomised trials. Lancet. 2002;359:1011-8.

24. Fine HA, Dear KB, Loeffler JS, Black PM, Canellos GP. Meta-analysis of radiation therapy with and without adjuvant chemotherapy for malignant gliomas in adults. Cancer. 1993;71:2585-97.

25. Stupp R, Wong ET, Kanner AA, Steinberg D, Engelhard H, Heidecke V, et al. Novo TTF-100A versus physician's choice chemotherapy in recurrent glioblastoma: A randomised phase III trial of a novel treatment modality. Eur J Cancer. 2012;48(14):2192-202.

\section{Submit your next manuscript to BioMed Central and take full advantage of:}

- Convenient online submission

- Thorough peer review

- No space constraints or color figure charges

- Immediate publication on acceptance

- Inclusion in PubMed, CAS, Scopus and Google Scholar

- Research which is freely available for redistribution 